\title{
$\begin{array}{ll}\text { Research Square } & \begin{array}{l}\text { Preprints are preliminary reports that have not undergone peer review. } \\ \text { They should not be considered conclusive, used to inform clinical practice, } \\ \text { or referenced by the media as validated information. }\end{array}\end{array}$
}

\section{Alleviating Effect of Quinoa and Underlying Mechanism on Hepatic Steatosis in High Fat Diet Fed Rat}

\section{Chenwei Song}

Shanghai JiaoTong University

Wei Lv

National Semi-Arid Agriculture Engineer Technology Research Center

Yahui Li

Center for Food Evaluation, State Administration for Market Regulation

Pan Nie

Shanghai JiaoTong University

Jun Lu

Chinese Academy of Sciences Shanghai Center for Plant Stress Biology

\section{Yanlou Geng}

National Semi-Arid Agriculture Engineering Technology Research Center

\section{Zhang Heng}

Chinese Academy of Sciences Shanghai Center for Plant Stress Biology

Lihua song ( $\square$ lihuas@sjtu.edu.cn )

Shanghai Jiao Tong University https://orcid.org/0000-0001-5549-4712

\section{Research}

Keywords: Quinoa, fatty liver, oxidative stress, lipids metabolism, metabonomics

Posted Date: October 11th, 2021

DOI: https://doi.org/10.21203/rs.3.rs-934267/v1

License: @ (i) This work is licensed under a Creative Commons Attribution 4.0 International License. Read Full License

Version of Record: A version of this preprint was published at Nutrition \& Metabolism on December 1st, 2021. See the published version at https://doi.org/10.1186/s12986-021-00631-7. 


\section{Abstract}

Background: HF diet-associated fatty liver (is also known as non-alcoholic fatty liver disease, NAFLD) is considered the hepatic component of the metabolic syndrome and has attracted widespread attention due to the increase in its prevalence. Daily dietary management, is considered to be one of the effective strategies for the prevention of NAFLD. In the present study, the effect of quinoa on the hepatic steatosis and the metabolism mechanism were investigated.

Methods: Male SD rats simultaneously administered an HF diet and different amounts of quinoa (equivalent to $100 \mathrm{~g} / \mathrm{day}$ and $300 \mathrm{~g} /$ day of human intake, respectively). After 12 weeks of the intervention, Hepatic TG and TC as well as serum anti-oxidative parameters were determined, H\&E staining evaluated the hepatic steatosis. Differential metabolite in serum and hepatic tissue were analyzed using UPLC-QTOF-MS ${ }^{\mathrm{E}}$. mRNA expression profile were investigated using RNA-Seq and further verified using real-time RT-PCR.

Results: It showed that quinoa effectively controlled the weight of rats, mitigated hepatic steatosis and oxidative stress, which exhibited the beneficial effect of quinoa on prevention of NAFLD. These beneficial effects could be attributed to the regulation of the production of certain metabolites in the circulation system or liver such as LysoPC and PC. The RNA-Seq analysis and RT-PCR verification revealed that an intake of a high amount of quinoa more effectively upregulated the genes related to lipid metabolism [Apoa (apolipoprotein)5, Apoa4, Apoc2) and down-regulated the genes related immune response [Irf (interferon regulatory factor) 5 , TIr6 (Toll like receptor), TIr10, TIr11, TIr12].

Conclusions: Quinoa could alleviate hepatic steatosis due to the regulation of metabolism and the expression of genes related with lipid metabolism and immune response.

\section{Introduction}

Nowadays, high-fat (HF) diet has become one of the common diet styles worldwide, resulting in metabolic health problems such as fatty liver, which is characterized by extravagant aggregation of triglycerides (TG) in the hepatocytes (hepatic steatosis). HF diet-associated fatty liver (is also known as nonalcoholic fatty liver disease, NAFLD) and is usually associated with hyperlipidemia, overweight/or obesity, and type 2 diabetes, and therefore is considered the hepatic component of the metabolic syndrome. Simple hepatic steatosis can progress to non-alcoholic steatohepatitis (NASH) and liver fibrosis, eventually causing cirrhosis and/or liver cancer if without any appropriate intervention [1]. Therefore, it has attracted widespread attention due to the increase in its prevalence from $20-41 \%$ worldwide [2], signifying its prevention and treatment as a strong public interest. However, there are currently no pharmaceutical interventions is approved for the treatment of NAFLD [3]. Lifestyle changes, especially daily dietary management, is considered to be one of the effective strategies [4] [5].

It is known that the primary factors that trigger hepatic steatosis is lipid overload. Accumulation of excessive fat causes hepatic lipotoxicity, which induces the liver cells to release pro-inflammatory cytokines, triggers oxidative stress, and activates hepatic stellate cells, ultimately leading to hepatic inflammatory injury. Improvement in lipid metabolism is an effective strategy for treating NAFLD [6]. Quinoa is a pseudo-cereal and abundant in proximate nutrients such as carbohydrates, high-quality proteins, dietary fibers, and microelements [7]. Moreover, quinoa is a rich source of bioactive phytochemicals such as polyphenols, flavonoids, rutins, and saponins [8] [9]. Among these, phenolic compounds and saponins in quinoa are phytochemicals that have attracted considerable attention of researchers [10]. A recent study reported that these phytochemicals of quinoa had potentially beneficial effects on human health. For example, Yao et al. [11] found that quinoa saponins inhibited the release of inflammatory cytokines, including tumor necrosis factor (TNF)-a and interleukin- 6 in lipopolysaccharide-induced RAW264.7 cells and suggested that quinoa saponins could be used as functional food components to prevent and treat inflammation. Other studies reported that phytochemicals in quinoa lowered the risk of oxidative stress-related diseases, e.g., cancer, cardiovascular diseases, diabetes, and obesity [12]. Especially, a clinical trial showed that the consumption of $50 \mathrm{~g}$ quinoa/day lowered the serum TGs in overweight and obese participants and reduced the prevalence of metabolic syndrome [13]. These beneficial effects of quinoa implied that it can prevent the progression of NAFLD. In fact, Mohamed et al. [14] reported that quinoa powder produced improvement in the biochemical parameters with different degrees in NAFLD rats. Similarly, Noratto et al. [15] demonstrated that quinoa intake reduced plasma and liver cholesterol, decreased obesity-associated inflammation, and prevented hepatic steatosis in obese $\mathrm{db} / \mathrm{db}$ mice. However, the action of quinoa intake on lipid metabolism of NAFLD and its related molecular mechanism still needs to be elucidated. In the present study, the effects of different amounts of quinoa on the metabolic profile of rats fed with HF diet were explored using non-target metabolomics, and the molecular mechanisms was investigated.

\section{Materials And Methods}

\subsection{Materials}

Quinoa is grown in the mountain dryland areas of Qinghai-Tibet Plateau (at an altitude of $4000 \mathrm{~m}$ ) and was provided by Qinghai Sanjiang Wotu Ecology Agriculture Technology Co., Ltd. (Qinghai, China). The assay kits for SOD (superoxide dismutase) (A001-3-2) and GSH-PX (glutathione peroxidase) (A005-1-2) activity, and the levels of MDA (malondialdehyde) (A003-1-2) and GSH (glutathione) (A006-1-1), TG (A110-2-1), and TC (total cholesterol) (A111-2-1) were purchased from the Jiancheng Biological (Nanjing, China). All other chemical reagents used were purchased from Sinopharm (Shanghai, China).

\subsection{Animals and experiment design}

Male Sprague-Dawley (SD) rats (specific pathogen-free [SPF] grade, 4 w, $100 \pm 10$ g) were provided by the Vital River Laboratory Animal Technology Co., Ltd. (Beijing, China).

Animals were maintained in a ventilated rack system. Food and water were provided ad libitum throughout the entire study. After a week of adaptive feeding, the rats were randomly allocated to the following four groups containing animals with similar mean body weights (BWs): a normal chow diet control group 
(NC, $n=7$ ), a high-fat diet group ( $\mathrm{HF}, n=7)$, a low-dose quinoa diet group (HF $+\mathrm{LQ}, n=7)$, and a high-dose quinoa diet group (HF $+\mathrm{HQ}, n=7)$. The rats in the NC group were fed a standard diet, and those in the other three groups were fed an HF diet. Simultaneously, the low-dose quinoa feed (HF + LQ) was added to $9 \%$ of quinoa (equivalent to $100 \mathrm{~g}$ of daily human intake), and the high-dose quinoa feed (HF $+\mathrm{HQ}$ ) was added to $27 \%$ of quinoa (equivalent to $300 \mathrm{~g}$ of daily human intake). The special feed was manufactured by FBSH Biotechnology Co. Ltd.

All rats were observed daily, and the BWs of all animals were recorded once a week. The experimental conditions and procedures were approved by the Shanghai Jiao Tong University Institutional Animal Care and Use Committee (A2018070).

\subsection{Sample collection}

After 12 weeks of the intervention, the rats were fasted overnight, weighed, and anesthetized with $2 \%$ sodium pentobarbital ( $0.2 \mathrm{~mL} / 100 \mathrm{~g})$ according to the recommendations for experimental animals. Blood samples were collected from the abdominal aorta, and serum was isolated by low-speed centrifugation $\left(3,509 \times \mathrm{g}, 10 \mathrm{~min}, 4^{\circ} \mathrm{C}\right)$. Livers were rapidly removed and weighed. The fresh liver samples from the same lobe and similar site in each rat were immediately fixed in $4 \%$ paraformaldehyde, and the other tissues were flash-frozen in liquid nitrogen. All samples were kept at $-80^{\circ} \mathrm{C}$ until further analysis.

\subsection{Histopathological analysis}

The liver tissues were fixed in $4 \%$ paraformaldehyde, processed, and embedded in paraffin for hematoxylin-eosin (H\&E) staining. The pathological section was observed and analyzed under an optical microscope (Olympus Soft Imaging Solutions GmbH, Münster, Germany).

\subsection{Determination of hepatic TG and TC}

Briefly, the fresh liver tissues ( $50 \mathrm{mg}$ of each sample) were extracted and homogenized using 10 times volume of ethanol. Then, the $10 \mu \mathrm{L}$ of supernatant was taken for determination after centrifugation. The levels of TG (GPO-PAP method) and TC (GPO-PAP method) in hepatic tissues were evaluated following the manufacturer's instructions.

\subsection{Analysis of anti-oxidative parameters}

The activities of serum SOD, GSH-Px, and CAT, and levels of GSH and MDA were evaluated according to the manufacturer's instructions. Briefly, the $50 \mu \mathrm{L}, 100$ $\mu \mathrm{L}, 300 \mu \mathrm{L}$ and $25 \mu \mathrm{L}$ of serum were used respectively for the determination of SOD and CAT activities as well as the GSH and MDA levels; For the measurement of GSH-PX, the $100 \mu \mathrm{L}$ of serum was used after 2 times dilution.

\subsection{Analysis of chemical composition of rat feed}

The crude protein content and fat content were determined using the Kjeldahl method (AOAC 2001.11) and the ether extract method (AOAC, 920.39), respectively. The content of crude fiber was analyzed using an AOAC method (AOAC, 962.09). The contents of ash (AOAC 942.05) and moisture (AOAC 2001.12) were measured using the gravimetric method. The content of carbohydrates in animal feeding was calculated according to the following minusing method: 100- content of moisture, ash, fat, protein and crude fibre.

2.8 Differential metabolite analysis in serum and hepatic tissue using UPLC-QTOF-MS ${ }^{\mathrm{E}}$ (ultraperformance liquid chromatography-quadrupole time-of-flight mass spectrometry)

For liver tissue, $50 \mathrm{mg}$ of freeze-dried liver powder was extracted with $500 \mu \mathrm{L}$ of methanol/acetonitrile/water $(1 / 1 / 1 ; \mathrm{v} / \mathrm{v} / \mathrm{v})$ by a homogenizer and incubated at $-20^{\circ} \mathrm{C}$ for $2 \mathrm{~h}$. The supernatant was collected for analysis after centrifugation $\left(12,000 \mathrm{rpm}, 4^{\circ} \mathrm{C}, 20 \mathrm{~min}\right)$.

For serum, $200 \mu \mathrm{L}$ of methanol/acetonitrile $(1 / 1 ; \mathrm{v} / \mathrm{v})$ was added to $50 \mu \mathrm{L}$ of serum, extracted at $-20^{\circ} \mathrm{C}$ for $2 \mathrm{~h}$, and vortexed every other $0.5 \mathrm{~h}$ during the extraction. The supernatant was collected for analysis after centrifugation $\left(12,000 \mathrm{rpm}, 4^{\circ} \mathrm{C}, 20 \mathrm{~min}\right)$.

Chromatography was performed with the chromatographic column, ACQUITY UPLC BEH C18 $(100 \times 2.1 \mathrm{~mm}, 1.7 \mu \mathrm{m}$; Waters Co., USA), and maintained at $45^{\circ} \mathrm{C}$. The gradient elution program for mobile phases $A(0.1 \%$ formic-water $)$ and $B(0.1 \%$ formic acid-acetonitrile) were as follows: $0-1$ min, $5-20 \% B ; 1-2.5$ min, $20-40 \%$ B; 2.5-9 min, 40-100\% B; 9-12 min, 100\% B; 12-12.5 min, 100-5\% B; $12.5-14.5 \mathrm{~min}, 5 \% \mathrm{~B}$; flow rate, $0.4 \mathrm{~mL} / \mathrm{min}$ and injection volume: $1 \mu \mathrm{L}$.

The ion source was operated in a positive and negative electrospray ionization (ESI) mode. The MS ${ }^{\mathrm{E}}$ scan mode alternated between low energy (CE 4 eV) and high energy (CE 20-45 eV) MSE modes. The scanning range was 50 to $1000 \mathrm{amu}$ and scanning speed maintained at $0.2 \mathrm{~s} / \mathrm{scan}$; capillary voltages were $1 \mathrm{kV}$ (negative mode) and $1.5 \mathrm{kV}$ (positive mode), cone voltage was $40 \mathrm{~V}$, source bias was $60 \mathrm{~V}$, source temperature was $115^{\circ} \mathrm{C}$, desolvation gas temperature was $450^{\circ} \mathrm{C}$, and the flow rate of the desolvation gas was $900 \mathrm{~L} / \mathrm{h}$, along with the cone gas flow rate of $50 \mathrm{~L} / \mathrm{h}$. The locking mass calibration Tyr-Gly-Phe-Leu (leucine-encephalin; $250 \mathrm{pg} / \mu \mathrm{L})$ was used as the lock mass $(\mathrm{m} / \mathrm{z} .554 .2620)$ for real-time calibration.

To ensure the consistence of analytical system, pooled quality control (QC) samples were made by mixing all samples. Ten QC samples were injected to equilibrate the system before analyzing. To evaluate the stability of the system, a QC sample was injected after every 10 sample injections during the analysis process.

\subsection{RNA-Seq}

Total RNA was extracted from fresh liver tissues (50 mg of each sample) using Trizol reagent (Invitrogen, USA). The cDNA libraries were constructed, sequenced, and analyzed by Huayou Biological Technology Co., Ltd. (Shanghai, China).

\subsection{RNA extraction and quantitative real-time PCR verification}


The first-strand cDNA was synthesized using the Prime-Script RT reagent kit (Takara Bio Inc., China). SYBR Premix Ex Taqll (Takara Bio Inc., China) was used in Quantitative real-time polymerase chain reaction (qRT-PCR). $\beta$-actin was used as endogenous references for mRNA expression. The comparative $2^{-} \triangle \triangle C t$ method was used to calculate the relative fold-change of target gene expression. The sequences of the primers in this study were showed in Supplementary Table 2.

\subsection{Data processing and statistical analysis}

Chromatographic and MS data (including the retention time, accurate mass, ion intensity, MS2 fragment) were collected and identified by Progenesis QI v2.3 (Waters Co., USA) based on an online database (MS/MS spectral database, HMDB [http://www.hmdb.ca/], LIPID MAPS [http://www.lipidmaps.org] and Metlin: https://metlin.scripps.edu/). Partial least squares discrimination analysis (PLS-DA) was performed to visually discriminate among the samples of different treatment groups. The differential metabolites were searched based on the variable weight values (VIP > 1), the smallest coefficient of variation (min. CV\% < $30 \%)$, and the $p$-value of the Student's $t$-test $(p<0.05)$.

All parameters are expressed as the mean \pm standard deviation (SD). The results were statistically analyzed by one-way analysis of variance (ANOVA) followed by the least significant difference (LSD) multiple comparison test. The criterion for significance was $p<0.05$.

\section{Results}

\subsection{Effect of quinoa diet on food intake and body weight of rat fed on a high-fat diet}

The macronutrient composition of feed for SD rats in each group were shown in Supplementary Table 1. The food intake of the NC group was remarkably higher $(p<0.01)$ than that of the three HF diet-fed groups, suggesting that an HF diet reduced the food intake probably due to higher calories than in the normal feed. However, the intake of feed containing quinoa, especially that of low-dose quinoa, was significantly higher than that of HF diet alone ( $p<0.01)$, implying that quinoa intake may improve the appetite of rats.

The average body weight (BW) of rats in the HF diet alone group was $630 \mathrm{~g}$ at the end of the experiment, which was significantly higher than that of rats in the $\mathrm{NC}$ group $(550 \mathrm{~g})(p<0.01)$, whereas the average BWs were $586 \mathrm{~g}$ and $604 \mathrm{~g}$ in the HF+LQ and HL+HQ groups, respectively. There was no significant difference compared with the NC group and two quinoa feeding groups (Fig. 1B).

\subsection{Effect of quinoa diet on hepatic pathological changes and lipid content of rats fed on HF diet}

A long-term HF diet can cause lipid deposition in the liver, resulting in insulin resistance, and further inducing metabolic disturbance to form a vicious cycle. We analyzed the hepatic histopathological changes in rats fed on an HF diet. HE-stained pathological sections of hepatic tissues are shown in Fig. $1 \mathrm{C}$. In the NC group, the hepatic lobules were clear, and hepatocytes were intact without fat accumulation. However, there were large lipid droplets, balloon-like degeneration, partial necrosis, multiple inflammatory aggregations, and severe steatosis of hepatocytes in the hepatic tissue of rats in the HF group. The hepatic intracellular lipid drop accumulation in the rats of the two quinoa diet groups was reduced as compared with those in the HF group. The levels of TG and TC in the hepatic tissue of rats in each group supported the histopathology results. The TC and TG contents of the HF, HF+LQ, and HF+HQ groups increased significantly $(p<0.01)$ compared with those of the NC group, whereas the TG $(p<0.05)$ and TC $(p<0.01)$ contents in the hepatic tissue of rats in the $\mathrm{HF}+\mathrm{LQ}$ and TC content $(p<0.01)$ in the hepatic tissue of rats in the HF+HQ groups were significantly lower than those of the HF group.

\subsection{Effects of quinoa diet on serum anti-oxidative parameters of rat fed on a high-fat diet}

The serum activities of SOD, GSH-Px, and levels of GSH and MDA in rats of each group are displayed in Fig. 2 A-D. The SOD and GSH-Px activities of rats fed on an $\mathrm{HF}$ diet alone were reduced by $23.8 \%$ and $41.3 \%$ as compared with those in the NC group $(p<0.01)$, while the SOD and GSH-PX activities of rats in the $\mathrm{HF}+\mathrm{LQ}$ and $\mathrm{HF}+\mathrm{HQ}$ groups were distinctly higher than those of rats in the HF group $((p<0.01)$ (Fig. $2 \mathrm{~A}, \mathrm{~B})$. The GSH levels of rats in the HF groups were slightly lower than those of rats in the $\mathrm{NC}, \mathrm{HF}+\mathrm{LQ}$, and $\mathrm{HF}+\mathrm{HQ}$ groups, although there were no significant differences among the four groups (Fig. $2 \mathrm{C}$ ).

MDA is a representative harmful lipid peroxide. The MDA levels in the rats of the HF group were significantly increased by $50 \%$, as compared to those in the NC group $(p<0.01)$, whereas the quinoa diet inhibited the increase in MDA levels as compared with the high fat diet alone group ( $p<0.01)$ (Fig. $2 \mathrm{D})$. High intake of quinoa increased the inhibition of the production of MDA as compared with that of low quinoa intake $(p<0.01)$ probably due to higher concentration of antioxidative phytochemicals. The above results suggested that quinoa effectively ameliorated the oxidative stress induced by an $\mathrm{HF}$ diet.

\subsection{Effects of quinoa diet on serum metabolites of rat fed on a high-fat diet}

Given the beneficial effects of quinoa on hepatic steatosis and oxidative stress in HF diet-fed rats, we subsequently investigated the serum metabolites using untargeted metabolomics. The score plots of each group showed distinct clustering, and the serum metabolite profile of rats in the quinoa diet group was different from that in the high fat diet alone groups (Fig. $3 \mathrm{~A}$ and B).

A total of 1,863 metabolites (ESI-, 691; ESI+, 1172) were detected by UPLC-Q-TOF-MS ${ }^{\mathrm{E}}$, and 46 differential serum metabolites are shown in Table 1. The differential metabolites were mainly included the related metabolites of PC (phosphatidylcholine), LysoPC (lysophosphatidylcholine), and PE (phosphatidylethanolamine), etc. (Fig. 3 C).

Specifically, in comparison with the HF group, low amount of quinoa (HF+LQ), significantly increased the levels of serum LysoPC (20:0, $p<0.05 ; 20: 1$ (11Z), $p<$ $0.01 ; 20: 2(11 Z, 14 Z), p<0.05)$; the high amount of quinoa (HF+HQ) increased the levels of SM (serum sphingomyelin) (18:0/24:1 (15Z), d d18:1/22:0, $p<$ 
0.01), gamma-linolenic acid, vitamin A, linoleic acid, myristic acid, Vitamin A and decreased the levels of PC (18:0/18:1 (11Z), 18:0/P-16:0) $(p<0.05)$ and PE (18:0/18:2 (9Z, 12Z)) (Fig. 3 D).

\subsection{Effects of quinoa diet on hepatic metabolites of rat fed on a high-fat diet}

Considering the important role of liver in lipids metabolism, we further investigated the hepatic metabolites. The score plots of each group exhibited distinct clustering, and the hepatic metabolite profile of rats in the quinoa diet group was different from that in the HF diet alone groups (Fig. 4 A and B).

A total of 1,295 (ESI-, 44; ESI+, 1251) were detected by UPLC-Q-TOF-MSE ${ }^{E}$, and 22 differential hepatic metabolites are shown in Table 2. Specifically, the quinoa diet, especially high amount of quinoa intake, significantly decreased the levels of $\mathrm{PC}(16: 0 / 18: 1$ (9Z), $p<0.05 ; \mathrm{PC}(18: 2$ (9Z, 12Z)/16:0), $p<0.01)$, dioleoylphosphatidylcholine $(p<0.01)$, and SM (d18:1/24:1(15Z)) $(p<0.01)$, and increased the levels of LysoPC $(20: 2(11 Z, 14 Z))$ and pantothenic acid $($ Fig. 4C) $(p<0.05)$, in comparison with the HF group.

\subsection{Effects of quinoa diet on the hepatic mRNA expression profile of rats fed on a high-fat diet}

To further investigate the mechanisms underlying the alleviating effects of quinoa on hepatic steatosis and its regulation of metabolism, we compared the transcriptome differences in the hepatic tissues between HF+LQ, HF+HQ, and HF groups (Fig. 5). Heatmap analysis exhibited the opposite expression profile comparing two quinoa diet intervention groups with HF diet alone group (Fig. $5 \mathrm{~A}$ ). Notably, compared with the HF group, gene ontology (GO) analysis revealed that the lower amount of quinoa (HF+LQ) mainly regulated the genes related to "positive regulation of bile acid biosynthetic process", "regulation of lipid metabolic process", indicating that the intake of quinoa diet (100 g/day) could influence the lipid metabolism (Fig. 5B). Intriguingly, the expression profile of the HF+HQ group was different from that of HF+LQ, when compared with that of the HF group. The regulated genes were mainly related to "positive regulation of triglyceride catabolic process", "cellular response to interleukin-1", "inflammatory response", "cellular response to tumor necrosis factor", "defense response to bacteria", "phospholipid efflux" and "negative regulation of toll-like receptor 4 signaling pathway". Besides, other genes were related to "cellular response to lipopolysaccharide", "positive regulation of lipoprotein lipase activity", "activated T cell proliferation", "positive regulation of the fatty acid biosynthetic process", "cellular response to interferon-gamma" and "response to oxidative stress", indicating that increased intake of quinoa more effectively regulated the genes related to lipid metabolism, immune response, and oxidative stress (Fig. 5B).

We further verified some of the mRNA expression using qRT-PCR. It showed that intakes of quinoa (especially HQ, equivalent to $300 \mathrm{~g}$ of daily human intake) could more effectively increase the expression levels of Apoa4 $(p<0.01)$, Apoa5 $(p<0.05)$ and Apoc2 $(p<0.01)$, which are genes related with lipids metabolic. Besides, high amount of quinoa could effectively decrease the expression levels of Irf (Interferon regulatory factor) $5, \mathrm{TIr}$ (Toll-like receptor) $6,10,11,12$ ( $p<$ 0.01) (Fig. 5C).

\section{Discussion}

Quinoa is a pseudo-grain with a high nutritional value because it is rich in proteins, lipids, fibers, vitamins, and minerals, and has an extraordinary balance of essential amino acids. In addition, quinoa possesses numerous secondary metabolites, such as phenolic acids, flavonoids, terpenoids, steroids, and nitrogencontaining compounds. These metabolites exhibit features beneficial to humans, including anti-diabetic [16], anticancer [17] [18], immunoregulatory [19], and adjuvant activities [20].

In the present study, the rats were administered an HF diet and different amounts of quinoa (equivalent to $100 \mathrm{~g} /$ day and $300 \mathrm{~g} /$ day of human intake) simultaneously. The results showed that low amounts of quinoa, which is equivalent to $100 \mathrm{~g} / \mathrm{d}$ of human intake, effectively controlled the weight of rats fed on an HF diet. Several studies have been investigated the effect of quinoa on weight. The vast majority of studies showed a positive association between quinoa consumption and decreased weight gain among animals [21]. This may contribute to the improvement of quinoa on hepatic steatosis. However, research investigating the health effects of different intakes of quinoa on the fatty liver and macular mechanism is scarce.

We found that intakes of quinoa $(100 \mathrm{~g} / \mathrm{d}$ and $300 \mathrm{~g} / \mathrm{d}$ ) reduced the TG and TC content in liver tissues, and mitigated pathological injury. Studies have reported that the consumption of quinoa was associated with decreased levels of serum cholesterol, TGs, low-density lipoprotein (LDL), and high-density lipoprotein (HDL) in rats fed on a high fructose diet [22]. Lin et al. [8] investigated the protective effects of quinoa bran extracts on CCI囚-induced liver injury and fibrosis in mice and found that red quinoa powder provided better protection than rutin against CCl囚-induced oxidative stress, pro-inflammatory factor expression, and fibrosis development. Noratto et al. [15] found that quinoa intake reduced the plasma and liver cholesterol, decreased obesity-associated inflammation, and prevented hepatic steatosis in obese $\mathrm{db} / \mathrm{db}$ mice, which partially exhibited consistency with our results. The above results implied that quinoa may exert positive role in the prevention of fatty liver.

Oxidative stress changes play a very important role in the pathogenesis and progression of NAFLD. Due to insulin resistance and excessive mobilization of peripheral fat in NAFLD patients, a large amount of free fatty acids can be produced, leading to $\beta$-oxidation overload and the imbalance of antioxidative system. This imbalance leads to lipid peroxidation, which can produce a large number of active and highly toxic intermediates, which eventually affect the normal function of liver cells[23]. In present study, we found that quinoa effectively improved the oxidative stress by increasing the activities of SOD and GSHPx, decreasing the levels of MDA under an HF diet. The antioxidant activity of quinoa has been previously investigated using several in vitro assays (such as the 2,2-diphenyl-1-picrylhydrazyl [DPPH] assay and ferric reducing antioxidant power [FRAP] assay) [24] [25] [26]. An in vivo study showed that the plasma lipid peroxidation reduced, whereas the expression of antioxidant enzymes such as GSH-Px and CAT were elevated in several organs [27]. The anti-oxidative activity of quinoa could be attributed to its abundant phytochemicals, such as saponin, phenolic acids, flavonoids, terpenoids, and steroids[28]. Among these, sapogenin is one of the major phytochemicals present abundantly in quinoa seeds and contribute to the anti-oxidative activity of quinoa [29]. These results further implied the beneficial role of quinoa in the prevention of NAFLD. 
Fatty liver has been associated with unfavorable metabolic changes in circulation [30]. To further elucidate the beneficial effects of quinoa on lipid metabolism regulation under an HF diet model, we investigated the serum and hepatic metabolic profiles of rats fed on an $\mathrm{HF}$ diet. It showed that quinoa intake increased the levels of partial LysoPC (20:0, 20:1, 20:2), SM (d 18:0/24:1, d18:1/22:0) and decreased those of PC (18:0/18:1, 18:0/P-16:0, 16:0/18:1, 18:2/16:0), etc. A recent clinical metabolomic/lipidomic-based analysis of plasma showed that levels of PC were positively correlated, and those of LysoPC were negatively correlated with hepatocellular ballooning in patients with NAFLD [31]. The metabolic profile of LysoPC and PC in the quinoa intake group may explain the alleviated effects on hepatic steatosis.

Interestingly, we found that high amounts of quinoa ( $\mathrm{HF}+\mathrm{HQ}$ ) increased the levels of serum $\mathrm{y}$-linolenic acid, linoleic acid, and vitamin A, and hepatic pantothenic acid. A study demonstrated that $Y$-linolenic acid could be a potential marker to predict the future development of metabolic syndrome (MS) in obese subjects [32]. Another study showed that a-linolenic acid-enriched perilla oil suppressed HF diet-induced hepatic steatosis by ameliorating the ER stressmediated autophagy [33]. Vitamin A is required for important physiological processes, including embryogenesis, vision, cell proliferation and differentiation, immune regulation, and glucose and lipid metabolism, while NAFLD may cause the disturbed Vitamin A metabolism [34]. A clinical survey on the relation between nutritional quality and NAFLD showed that healthy subjects had a higher index of nutritional quality (INQ) of pantothenic acid and other vitamins, including vitamin D, vitamin E, and biotin, compared to patients with NAFLD [35]. Therefore, the above metabolic profile may elucidate the protective effects of quinoa against the progression of NAFLD. However, the specific molecular mechanism still needs further exploration.

To further investigate the molecular mechanism of quinoa on metabolism, we explored the mRNA expression profile of hepatic tissue. It showed that the low amount quinoa intakes could regulated the genes related to lipid metabolism; while high amount of quinoa intakes mainly regulated the genes related to lipid metabolism and immune response. Specifically, quinoa intakes could significantly up-regulated the mRNA expression levels of apolipoprotein including Apoa4, Apoa5 and Apoc2, which are known to augment lipoprotein lipase (LPL) activity [36]. LPL is bound to the vascular endothelium, and hydrolyzes chylomicron and VLDL-associated TG to facilitate the transport of hydrolyzed fatty acids to peripheral cells. Patients with genetic defects in Apoc2, Apoa5 or LPL display high circulating TG levels due to impaired TG clearance [36] [37] [38]. Moreover, quinoa intakes could significantly down-regulated the mRNA expression levels of Irf5, TIr 6, 10,11, 12. A recent study showed that elevated adipose Irf 5 expression in obesity concurs with the typical inflammatory signatures, locally and systemically. The upregulation of Irf5 may represent a novel adipose tissue marker for metabolic inflammation [39]. Gut microbiota is a source of Toll-like receptor (TLR) ligands, which can stimulate liver cells to produce proinflammatory cytokines under certain conditions. However, the liver has a high tolerance to TLR ligands because hepatic cells express minimal TLRs in normal liver. In contrast, TLR signaling is activated and downstream molecules are increased in NAFLD because the tolerance has been disrupted [40]. Indeed, hepatic TLR expression are increased in human NAFLD patients as well as in animal models [41] [42] [43] [44]. To date, TIr2, 4, 5, and TIr9 have been shown to be associated with the pathogenesis of NAFLD [45].Thus, it is suggested that TLRs are potential targets for NAFLD treatment. The above results primarily unveiled the molecular mechanism of benefits of quinoa on NAFLD.

\section{Conclusion}

In summary, our results demonstrated the beneficial effects of quinoa on weight gain control and improving hepatic steatosis and oxidative stress state under the HF diet style. These beneficial effects could be attributed to the regulation of the production of certain metabolites in the circulation system and the liver, including linoleic acid, vitamin A and the content of partial lipid metabolites such as lysophosphatidylcholine (LysoPC) and PC. Findings of the present study suggest the positive role quinoa in lowering risk of NAFLD. The RNA-seq analysis results primarily elucidated that high amounts of quinoa intake more effectively enriched the gene sets involved in lipids metabolism and immune response, especially the regulation action on partial lipids metabolic genes and Toll like receptors. However, more research is still needed on the investigation of signaling pathway of those genes involved in.

\section{Abbreviations}

NAFLD: Nonalcoholic fatty liver disease; NASH: non-alcoholic steatohepatitis; LysoPC: lysophosphatidylcholine; PC: phosphatidylcholine; PE: phosphatidylethanolamine; SM: serum sphingomyelin; Apoa: apolipoprotein; Irf5: interferon regulatory factor; Tlr: Toll like receptor; TG: triglycerides; TC: total cholesterol; SOD: superoxide dismutase; GSH-PX: glutathione peroxidase; MDA: malondialdehyde; GSH: glutathione; UPLC/Q-TOF-MS: ultraperformance liquid chromatography-quadrupole time-of-flight mass spectrometry

\section{Declarations}

\section{Acknowledgements}

We thank our colleagues for their association and helpful advices in this study.

\section{Authors' contributions}

Planning of the study was performed with lihua Song, Heng Zhang and Yanlou Geng. Conducting of the study was performed with Chenwei Song and Pan Nie. Collecting of the data was carried out by Pan Nie and Jun Lu. Interpretation of data was carried out by Chenwei Song, Wei Lv and Yahui Li. Drafting the manuscript was performed by Chenwei Song and revised by Lihua Song. All authors have approved the final draft and agreed to submit manuscript to this journal.

\section{Funding}

This work was supported by grants from Key Technology Research and Development Program of Hebei Province (CN) (19227527D), Shanghai Agriculture Applied Technology Development Program (2019-02-08-00-08-F01154) and Oceanic Interdisciplinary Program of Shanghai Jiao Tong University 
(SL2020ZD103).

Availability of data and materials

The data and materials that support the findings of this study are available from the corresponding author upon reasonable request.

Ethics approval and consent to participate

The protocols of this study were approved by the Institutional Animal Care and Use Committee of Shanghai JiaoTong University.

Consent for publication

All authors consent to the publication of the manuscript.

Competing interests

The authors declare that there is no competing of interest.

\section{References}

1. Rahimi RS, Landaverde C: Nonalcoholic fatty liver disease and the metabolic syndrome: clinical implications and treatment.Nutr Clin Pract 2013, 28:40-51.

2. Hsu WF, Sheen LY, Lin HJ, Chang HH: A Review of Western and Traditional Chinese Medical Approaches to Managing Nonalcoholic Fatty Liver Disease.Evid Based Complement Alternat Med 2016, 2016:6491420.

3. Mundi MS, Velapati S, Patel J, Kellogg TA, Abu Dayyeh BK, Hurt RT: Evolution of NAFLD and Its Management.Nutr Clin Pract 2020, 35:72-84.

4. Romero-Gomez M, Zelber-Sagi S, Trenell M: Treatment of NAFLD with diet, physical activity and exercise.J Hepato/ 2017, 67:829-846.

5. Perdomo CM, Fruhbeck G, Escalada J: Impact of Nutritional Changes on Nonalcoholic Fatty Liver Disease.Nutrients $2019,11$.

6. Xu Y, Guo W, Zhang C, Chen F, Tan HY, Li S, Wang N, Feng Y: Herbal Medicine in the Treatment of Non-Alcoholic Fatty Liver Diseases-Efficacy, Action Mechanism, and Clinical Application.Front Pharmacol 2020, 11:601.

7. Vega-Galvez A, Miranda M, Vergara J, Uribe E, Puente L, Martinez EA: Nutrition facts and functional potential of quinoa (Chenopodium quinoa willd.), an ancient Andean grain: a review.J Sci Food Agric 2010, 90:2541-2547.

8. Lin TA, Ke BJ, Cheng CS, Wang JJ, Wei BL, Lee CL: Red Quinoa Bran Extracts Protects against Carbon Tetrachloride-Induced Liver Injury and Fibrosis in Mice via Activation of Antioxidative Enzyme Systems and Blocking TGF-beta1 Pathway.Nutrients 2019, 11.

9. Martinez-Villaluenga C, Penas E, Hernandez-Ledesma B: Pseudocereal grains: Nutritional value, health benefits and current applications for the development of gluten-free foods. Food Chem Toxicol 2020, 137:111178.

10. Gomez-Caravaca AM, Segura-Carretero A, Fernandez-Gutierrez A, Caboni MF: Simultaneous determination of phenolic compounds and saponins in quinoa (Chenopodium quinoa Willd) by a liquid chromatography-diode array detection-electrospray ionization-time-of-flight mass spectrometry methodology.J Agric Food Chem 2011, 59:10815-10825.

11. Yao Y, Yang X, Shi Z, Ren G: Anti-inflammatory activity of saponins from quinoa (Chenopodium quinoa Willd.) seeds in lipopolysaccharide-stimulated RAW 264.7 macrophages cells.J Food Sci 2014, 79:H1018-1023.

12. Tang Y, Tsao R: Phytochemicals in quinoa and amaranth grains and their antioxidant, anti-inflammatory, and potential health beneficial effects: a review.Mol Nutr Food Res 2017, 61.

13. Navarro-Perez D, Radcliffe J, Tierney A, Jois M: Quinoa Seed Lowers Serum Triglycerides in Overweight and Obese Subjects: A Dose-Response Randomized Controlled Clinical Trial.Curr Dev Nutr 2017, 1:e001321.

14. Mohamed DA, Fouda KA, Mohamed RS: <i>In vitro</i> Anticancer Activity of Quinoa and Safflower Seeds and Their Preventive Effects on Non-alcoholic Fatty Liver.Pak J Biol Sci 2019, 22:383-392.

15. Noratto GD, Murphy K, Chew BP: Quinoa intake reduces plasma and liver cholesterol, lessens obesity-associated inflammation, and helps to prevent hepatic steatosis in obese db/db mouse.Food Chem 2019, 287:107-114.

16. Graf BL, Poulev A, Kuhn P, Grace MH, Lila MA, Raskin I: Quinoa seeds leach phytoecdysteroids and other compounds with anti-diabetic properties.Food Chem 2014, 163:178-185.

17. Hu Y, Zhang J, Zou L, Fu C, Li P, Zhao G: Chemical characterization, antioxidant, immune-regulating and anticancer activities of a novel bioactive polysaccharide from Chenopodium quinoa seeds.Int J Biol Macromol 2017, 99:622-629.

18. Kuljanabhagavad T, Thongphasuk P, Chamulitrat W, Wink M: Triterpene saponins from Chenopodium quinoa Willd.Phytochemistry 2008, 69:1919-1926.

Page 7/16 
19. Yao Y, Shi Z, Ren G: Antioxidant and immunoregulatory activity of polysaccharides from quinoa (Chenopodium quinoa Willd.).Int $J$ Mol Sci 2014 , 15:19307-19318.

20. Estrada A, Li B, Laarveld B: Adjuvant action of Chenopodium quinoa saponins on the induction of antibody responses to intragastric and intranasal administered antigens in mice.Comp Immunol Microbiol Infect Dis 1998, 21:225-236.

21. Simnadis TG, Tapsell LC, Beck EJ: Physiological Effects Associated with Quinoa Consumption and Implications for Research Involving Humans: a Review.Plant Foods Hum Nutr 2015, 70:238-249.

22. Pasko P, Zagrodzki P, Barton H, Chlopicka J, Gorinstein S: Effect of quinoa seeds (Chenopodium quinoa) in diet on some biochemical parameters and essential elements in blood of high fructose-fed rats.Plant Foods Hum Nutr 2010, 65:333-338.

23. Swiderska M, Maciejczyk M, Zalewska A, Pogorzelska J, Flisiak R, Chabowski A: Oxidative stress biomarkers in the serum and plasma of patients with non-alcoholic fatty liver disease (NAFLD). Can plasma AGE be a marker of NAFLD? Oxidative stress biomarkers in NAFLD patients.Free Radic Res 2019, 53:841-850.

24. Park JH, Lee YJ, Kim YH, Yoon KS: Antioxidant and Antimicrobial Activities of Quinoa (Chenopodium quinoa Willd.) Seeds Cultivated in Korea.Prev Nutr Food Sci 2017, 22:195-202.

25. Tang Y, Li X, Zhang B, Chen PX, Liu R, Tsao R: Characterisation of phenolics, betanins and antioxidant activities in seeds of three Chenopodium quinoa Willd. genotypes.Food Chem 2015, 166:380-388.

26. Ong ES, Pek CJN, Tan JCW, Leo CH: Antioxidant and Cytoprotective Effect of Quinoa (Chenopodium quinoa Willd.) with Pressurized Hot Water Extraction (PHWE).Antioxidants (Basel) 2020, 9.

27. Pasko P, Barton H, Zagrodzki P, Izewska A, Krosniak M, Gawlik M, Gawlik M, Gorinstein S: Effect of diet supplemented with quinoa seeds on oxidative status in plasma and selected tissues of high fructose-fed rats.Plant Foods Hum Nutr 2010, 65:146-151.

28. Lin M, Han P, Li Y, Wang W, Lai D, Zhou L: Quinoa Secondary Metabolites and Their Biological Activities or Functions.Molecules $2019,24$.

29. Lim JG, Park HM, Yoon KS: Analysis of saponin composition and comparison of the antioxidant activity of various parts of the quinoa plant (Chenopodium quinoa Willd.).Food Sci Nutr 2020, 8:694-702.

30. Sliz E, Sebert S, Wurtz P, Kangas AJ, Soininen P, Lehtimaki T, Kahonen M, Viikari J, Mannikko M, Ala-Korpela M, et al: NAFLD risk alleles in PNPLA3, TM6SF2, GCKR and LYPLAL1 show divergent metabolic effects. Hum Mol Genet 2018, 27:2214-2223.

31. Ogawa Y, Kobayashi T, Honda Y, Kessoku T, Tomeno W, Imajo K, Nakahara T, Oeda S, Nagaoki Y, Amano Y, et al: Metabolomic/lipidomic-based analysis of plasma to diagnose hepatocellular ballooning in patients with non-alcoholic fatty liver disease: A multicenter study. Hepatol Res 2020, 50:955-965.

32. Ni Y, Zhao L, Yu H, Ma X, Bao Y, Rajani C, Loo LW, Shvetsov YB, Yu H, Chen T, et al: Circulating Unsaturated Fatty Acids Delineate the Metabolic Status of Obese Individuals. EBioMedicine 2015, 2:1513-1522.

33. Bae SJ, Kim JE, Choi HJ, Choi YJ, Lee SJ, Gong JE, Seo S, Yang SY, An BS, Lee HS, et al: alpha-Linolenic Acid-Enriched Cold-Pressed Perilla Oil Suppress High-Fat Diet-Induced Hepatic Steatosis through Amelioration of the ER Stress-Mediated Autophagy.Molecules 2020, 25.

34. Saeed A, Dullaart RPF, Schreuder T, Blokzijl H, Faber KN: Disturbed Vitamin A Metabolism in Non-Alcoholic Fatty Liver Disease (NAFLD).Nutrients 2017, 10.

35. Vahid F, Hekmatdoost A, Mirmajidi S, Doaei S, Rahmani D, Faghfoori Z: Association Between Index of Nutritional Quality and Nonalcoholic Fatty Liver Disease: The Role of Vitamin D and B Group.Am J Med Sci 2019, 358:212-218.

36. Marcais C, Verges B, Charriere S, Pruneta V, Merlin M, Billon S, Perrot L, Drai J, Sassolas A, Pennacchio LA, et al: Apoa5 Q139X truncation predisposes to late-onset hyperchylomicronemia due to lipoprotein lipase impaiment.J Clin Invest 2005, 115:2862-2869.

37. Merkel M, Eckel RH, Goldberg IJ: Lipoprotein lipase: genetics, lipid uptake, and regulation.J Lipid Res 2002, 43:1997-2006.

38. Pennacchio LA, Olivier M, Hubacek JA, Cohen JC, Cox DR, Fruchart JC, Krauss RM, Rubin EM: An apolipoprotein influencing triglycerides in humans and mice revealed by comparative sequencing.Science 2001, 294:169-173.

39. Sindhu S, Thomas R, Kochumon S, Wilson A, Abu-Farha M, Bennakhi A, Al-Mulla F, Ahmad R: Increased Adipose Tissue Expression of Interferon Regulatory Factor (IRF)-5 in Obesity: Association with Metabolic Inflammation.Cells 2019, 8.

40. Miyake Y, Yamamoto K: Role of gut microbiota in liver diseases. Hepatol Res 2013, 43:139-146.

41. Tsukumo DM, Carvalho-Filho MA, Carvalheira JB, Prada PO, Hirabara SM, Schenka AA, Araujo EP, Vassallo J, Curi R, Velloso LA, Saad MJ: Loss-of-function mutation in Toll-like receptor 4 prevents diet-induced obesity and insulin resistance.Diabetes 2007, 56:1986-1998.

42. Rivera CA, Adegboyega P, van Rooijen N, Tagalicud A, Allman M, Wallace M: Toll-like receptor-4 signaling and Kupffer cells play pivotal roles in the pathogenesis of non-alcoholic steatohepatitis.J Hepatol 2007, 47:571-579.

Page $8 / 16$ 
43. Spruss A, Kanuri G, Wagnerberger S, Haub S, Bischoff SC, Bergheim I: Toll-like receptor 4 is involved in the development of fructose-induced hepatic steatosis in mice.Hepatology 2009, 50:1094-1104.

44. Kodama Y, Kisseleva T, Iwaisako K, Miura K, Taura K, De Minicis S, Osterreicher CH, Schnabl B, Seki E, Brenner DA: c-Jun N-terminal kinase-1 from hematopoietic cells mediates progression from hepatic steatosis to steatohepatitis and fibrosis in mice.Gastroenterology 2009, 137:1467-1477 e1465.

45. Miura K, Ohnishi H: Role of gut microbiota and Toll-like receptors in nonalcoholic fatty liver disease. World J Gastroenterol 2014, 20:7381-7391.

\section{Tables}

Table 1. Identification of differential metabolites in serum of rats in each group

Table 2. Identification of differential metabolites in the liver of rats in each group

PC: phosphatidylcholine; LysoPC: lysophosphatidylcholine; SM: sphingomyelin; FAD: flavin adenine dinucleotide

\section{Figures}

A
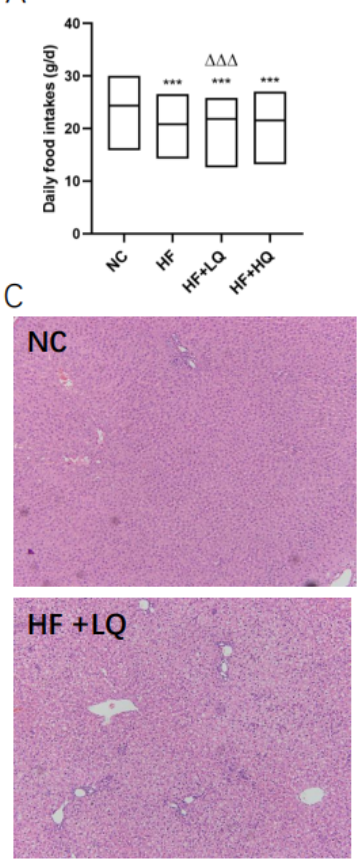

D

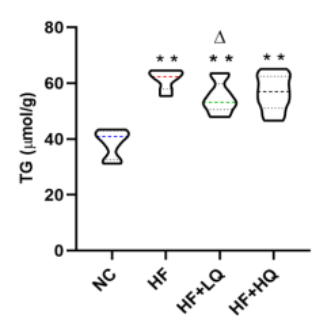

B
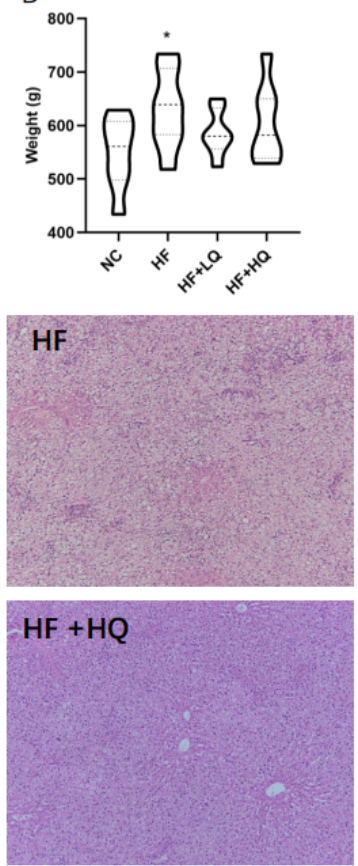

$\mathrm{E}$

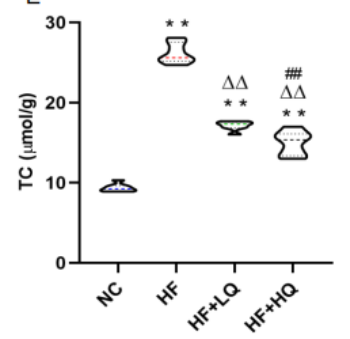

\section{Figure 1}

Effects of quinoa on daily food intake, weight and hepatic steatosis histology changes of rats fed on a high-fat diet. A. Daily food intake. B. Weight. C. Hepatic histology. D. Hepatic TG content. E. Hepatic TC content. NC: normal chow diet control group ( $n=7$ ); HF: a high-fat diet group ( $n=7$ ); HF + LQ: low-dose quinoa diet group ( $n=7)$; HF + HQ: a high-dose quinoa diet group $(n=7)$. The rats in the NC group were fed a standard diet, and those in the other three groups were fed an HF diet. Simultaneously, the low-dose quinoa feed (HF + LQ) was added to $9 \%$ of quinoa (equivalent to $100 \mathrm{~g}$ of daily human intake), and the high-dose quinoa feed ( $\mathrm{HF}+\mathrm{HQ}$ ) was added to $27 \%$ of quinoa (equivalent to $300 \mathrm{~g}$ of daily human intake). ${ }^{\star} \mathrm{p}<0.05 \mathrm{vs}$. the NC group, ${ }^{\star *} \mathrm{p}<0.01 \mathrm{vs}$. the NC group; $\Delta \mathrm{p}<$ 0.05 vs. the HF group, $\Delta \Delta \mathrm{p}<0.01$ vs. the HF group; \#p $<0.05$ vs. the HF + LQ group; \#\#p $<0.01$ vs. the HF + LQ group. The bars show the mean values with standard deviations. 


\begin{tabular}{|c|c|c|c|c|c|c|c|c|c|}
\hline \multirow[t]{2}{*}{ Component name } & \multirow{2}{*}{$\begin{array}{l}\text { MS } \\
(m / z)\end{array}$} & \multirow{2}{*}{$\begin{array}{l}\text { Observed } \\
\text { RT (min) }\end{array}$} & \multirow{2}{*}{$\begin{array}{l}\text { Mass } \\
\text { error } \\
(\mathrm{ppm})\end{array}$} & \multirow[t]{2}{*}{ Formula } & \multirow[t]{2}{*}{ Adducts } & \multirow[t]{2}{*}{ VIP } & \multicolumn{3}{|c|}{ Trend } \\
\hline & & & & & & & $\mathrm{NC} / \mathrm{HF}$ & $\mathrm{HF}+\mathrm{LQ} / \mathrm{HF}$ & $\mathrm{HF}+\mathrm{HQ} /]$ \\
\hline SM(d18:0/24:1(15Z)) & 837.6820 & 10.45 & -0.4 & $\mathrm{C}_{47} \mathrm{H}_{95} \mathrm{~N}_{2} \mathrm{O}_{6} \mathrm{P}$ & $\begin{array}{l}\mathrm{M}+\mathrm{H} \\
\mathrm{M}+\mathrm{Na}\end{array}$ & 1.4 & $\uparrow$ & $\uparrow$ & $\uparrow$ \\
\hline $\operatorname{SM}(d 18: 1 / 22: 0)$ & 809.6501 & 10.08 & -0.5 & $\mathrm{C}_{45} \mathrm{H}_{91} \mathrm{~N}_{2} \mathrm{O}_{6} \mathrm{P}$ & $\begin{array}{l}\mathrm{M}+\mathrm{H} \\
\mathrm{M}+\mathrm{Na}\end{array}$ & 2.5 & $\uparrow$ & $\uparrow$ & $\uparrow$ \\
\hline $\operatorname{SM}(d 18: 1 / 16: 0)$ & 703.5745 & 8.94 & -2.4 & $\mathrm{C}_{39} \mathrm{H}_{79} \mathrm{~N}_{2} \mathrm{O}_{6} \mathrm{P}$ & $\begin{array}{c}\mathrm{M}+\mathrm{H}- \\
\mathrm{H}_{2} \mathrm{O} \\
\mathrm{M}+\mathrm{H}\end{array}$ & 4.7 & $\uparrow$ & $\uparrow$ & $\downarrow$ \\
\hline $\mathrm{PC}(16: 0 / 20: 3(8 Z, 11 \mathrm{Z}, 14 \mathrm{Z}))$ & 784.5844 & 8.95 & -0.8 & $\mathrm{C}_{44} \mathrm{H}_{82} \mathrm{NO}_{8} \mathrm{P}$ & $\begin{array}{l}\mathrm{M}+\mathrm{H} \\
\mathrm{M}+\mathrm{Na}\end{array}$ & 4.0 & $\downarrow$ & $\uparrow$ & $\uparrow$ \\
\hline PC(16:0/22:5(7Z,10Z,13Z,16Z,19Z)) & 852.5746 & 8.77 & -1.8 & $\mathrm{C}_{46} \mathrm{H}_{82} \mathrm{NO}_{8} \mathrm{P}$ & $\begin{array}{c}\mathrm{M}+\mathrm{FA}- \\
\mathrm{H}\end{array}$ & 1.0 & $\uparrow$ & $\uparrow$ & $\downarrow$ \\
\hline $\mathrm{PC}(16: 1(9 Z) / 20: 4(8 Z, 11 Z, 14 Z, 17 Z))$ & 780.5532 & 8.49 & -0.7 & $\mathrm{C}_{44} \mathrm{H}_{78} \mathrm{NO}_{8} \mathrm{P}$ & $\begin{array}{l}\mathrm{M}+\mathrm{H} \\
\mathrm{M}+\mathrm{Na}\end{array}$ & 1.6 & $\uparrow$ & $\downarrow$ & $\uparrow$ \\
\hline $\mathrm{PC}(18: 1(9 \mathrm{Z}) / 18: 1(9 \mathrm{Z}))$ & 808.5833 & 8.78 & 0.8 & $\mathrm{C}_{44} \mathrm{H}_{84} \mathrm{NO}_{8} \mathrm{P}$ & $\mathrm{M}+\mathrm{Na}$ & 2.7 & $\uparrow$ & $\uparrow$ & $\uparrow$ \\
\hline $\mathrm{PC}(18: 0 / 18: 1(9 \mathrm{Z}))$ & 788.6158 & 9.52 & -0.7 & $\mathrm{C}_{44} \mathrm{H}_{86} \mathrm{NO}_{8} \mathrm{P}$ & $\mathrm{M}+\mathrm{H}$ & 3.0 & $\downarrow$ & $\downarrow$ & $\downarrow$ \\
\hline $\mathrm{PC}(18: 0 / 16: 0)$ & 762.6001 & 9.54 & -0.8 & $\mathrm{C}_{42} \mathrm{H}_{84} \mathrm{NO}_{8} \mathrm{P}$ & $\mathrm{M}+\mathrm{H}$ & 1.0 & $\downarrow$ & $\downarrow$ & $\downarrow$ \\
\hline $\mathrm{PC}(18: 0 / 22: 6(4 Z, 7 Z, 10 Z, 13 Z, 16 Z, 19 Z))$ & 834.5998 & 8.98 & -1.1 & $\mathrm{C}_{48} \mathrm{H}_{84} \mathrm{NO}_{8} \mathrm{P}$ & $\begin{array}{l}\mathrm{M}+\mathrm{H} \\
\mathrm{M}+\mathrm{Na}\end{array}$ & 5.4 & $\uparrow$ & $\downarrow$ & $\downarrow$ \\
\hline $\mathrm{PC}(18: 0 / 20: 3(5 Z, 8 Z, 11 Z))$ & 812.6154 & 9.27 & -1.2 & $\mathrm{C}_{46} \mathrm{H}_{86} \mathrm{NO}_{8} \mathrm{P}$ & $\mathrm{M}+\mathrm{H}$ & 3.1 & $\downarrow$ & $\downarrow$ & $\downarrow$ \\
\hline $\mathrm{PC}(18: 0 / 18: 2(9 \mathrm{Z}, 12 \mathrm{Z}))$ & 786.6002 & 9.22 & -0.7 & $\mathrm{C}_{44} \mathrm{H}_{84} \mathrm{NO}_{8} \mathrm{P}$ & $\mathrm{M}+\mathrm{H}$ & 4.8 & $\downarrow$ & $\downarrow$ & $\downarrow$ \\
\hline $\mathrm{PC}(18: 2(9 \mathrm{Z}, 12 \mathrm{Z}) / 20: 4(5 \mathrm{Z}, 8 \mathrm{Z}, 11 \mathrm{Z}, 14 \mathrm{Z}))$ & 828.5507 & 8.52 & -0.9 & $\mathrm{C}_{46} \mathrm{H}_{80} \mathrm{NO}_{8} \mathrm{P}$ & $\begin{array}{l}\mathrm{M}+\mathrm{H} \\
\mathrm{M}+\mathrm{Na}\end{array}$ & 3.5 & $\uparrow$ & $\downarrow$ & $\uparrow$ \\
\hline $\mathrm{PC}(18: 0 / \mathrm{P}-16: 0)$ & 768.5872 & 9.40 & -0.7 & $\mathrm{C}_{42} \mathrm{H}_{84} \mathrm{NO}_{7} \mathrm{P}$ & $\begin{array}{l}\mathrm{M}+\mathrm{H} \\
\mathrm{M}+\mathrm{Na}\end{array}$ & 3.2 & $\downarrow$ & $\downarrow$ & $\downarrow$ \\
\hline $\mathrm{PC}(18: 0 / 18: 1(11 \mathrm{Z}))$ & 832.6059 & 9.53 & -1.9 & $\mathrm{C}_{44} \mathrm{H}_{86} \mathrm{NO}_{8} \mathrm{P}$ & $\begin{array}{c}\mathrm{M}+\mathrm{FA}- \\
\mathrm{H}\end{array}$ & 1.6 & $\downarrow$ & $\downarrow$ & $\downarrow$ \\
\hline $\mathrm{PC}(20: 3(5 \mathrm{Z}, 8 \mathrm{Z}, 11 \mathrm{Z}) / 16: 0)$ & 806.5687 & 8.67 & 2.1 & $\mathrm{C}_{44} \mathrm{H}_{82} \mathrm{NO}_{8} \mathrm{P}$ & $\mathrm{M}+\mathrm{Na}$ & 4.9 & $\uparrow$ & $\downarrow$ & $\downarrow$ \\
\hline $\mathrm{PC}(20: 2(11 \mathrm{Z}, 14 \mathrm{Z}) / 18: 0)$ & 836.6147 & 9.09 & 0.9 & $\mathrm{C}_{46} \mathrm{H}_{88} \mathrm{NO}_{8} \mathrm{P}$ & $\mathrm{M}+\mathrm{Na}$ & 1.1 & $\uparrow$ & $\uparrow$ & $\downarrow$ \\
\hline PC(20:4(5Z,8Z,11Z,14Z)/22:6(4Z,7Z,10Z,13Z,16Z,19Z)) & 854.5694 & 8.33 & 0.0 & $\mathrm{C}_{50} \mathrm{H} 80 \mathrm{NO} 8 \mathrm{P}$ & $\begin{array}{l}\mathrm{M}+\mathrm{H} \\
\mathrm{M}+\mathrm{Na}\end{array}$ & 1.1 & $\uparrow$ & $\downarrow$ & $\downarrow$ \\
\hline $\mathrm{PE}(18: 0 / 18: 2(9 \mathrm{Z}, 12 \mathrm{Z}))$ & 742.5386 & 8.90 & -0.8 & $\mathrm{C}_{41} \mathrm{H}_{78} \mathrm{NO}_{8} \mathrm{P}$ & $\mathrm{M}-\mathrm{H}$ & 1.0 & $\downarrow$ & $\downarrow$ & $\downarrow$ \\
\hline PE(22:5(4Z,7Z,10Z,13Z,16Z)/18:1 (11Z)) & 790.5392 & 8.68 & 0.0 & $\mathrm{C}_{45} \mathrm{H}_{78} \mathrm{NO}_{8} \mathrm{P}$ & $\mathrm{M}-\mathrm{H}$ & 1.1 & $\uparrow$ & $\downarrow$ & $\downarrow$ \\
\hline LysoPC(16:0) & 496.3397 & 6.03 & -0.2 & $\mathrm{C}_{24} \mathrm{H}_{50} \mathrm{NO}_{7} \mathrm{P}$ & $\begin{array}{c}\mathrm{M}+\mathrm{H}- \\
\mathrm{H}_{2} \mathrm{O} \\
\mathrm{M}+\mathrm{H} \\
2 \mathrm{M}+\mathrm{H}\end{array}$ & 6.8 & $\uparrow$ & $\uparrow$ & $\downarrow$ \\
\hline LysoPC(15:0) & 482.3238 & 5.79 & -0.7 & $\mathrm{C}_{23} \mathrm{H}_{48} \mathrm{NO}_{7} \mathrm{P}$ & $\begin{array}{l}\mathrm{M}+\mathrm{H}- \\
\mathrm{H}_{2} \mathrm{O} \\
\mathrm{M}+\mathrm{H} \\
\mathrm{M}+\mathrm{Na}\end{array}$ & 2.2 & $\uparrow$ & $\uparrow$ & $\downarrow$ \\
\hline LysoPC(14:0) & 468.3081 & 5.32 & -0.4 & $\mathrm{C}_{22} \mathrm{H}_{46} \mathrm{NO}_{7} \mathrm{P}$ & $\begin{array}{c}\mathrm{M}+\mathrm{H}- \\
\mathrm{H}_{2} \mathrm{O} \\
\mathrm{M}+\mathrm{H} \\
\mathrm{M}+\mathrm{Na}\end{array}$ & 2.2 & $\uparrow$ & $\uparrow$ & $\uparrow$ \\
\hline LysoPC(16:1(9Z)) & 494.3238 & 5.52 & -0.7 & $\mathrm{C}_{24} \mathrm{H}_{48} \mathrm{NO}_{7} \mathrm{P}$ & $\begin{array}{c}\mathrm{M}+\mathrm{H}- \\
\mathrm{H}_{2} \mathrm{O} \\
\mathrm{M}+\mathrm{H} \\
\mathrm{M}+\mathrm{Na}\end{array}$ & 3.8 & $\uparrow$ & $\uparrow$ & $\uparrow$ \\
\hline LysoPC(20:0) & 552.4022 & 7.51 & 1.0 & $\mathrm{C}_{28} \mathrm{H}_{58} \mathrm{NO}_{7} \mathrm{P}$ & $\begin{array}{c}\mathrm{M}+\mathrm{H}- \\
\mathrm{H}_{2} \mathrm{O} \\
\mathrm{M}+\mathrm{H} \\
\mathrm{M}+\mathrm{Na}\end{array}$ & 1.4 & $\uparrow$ & $\uparrow$ & $\uparrow$ \\
\hline LysoPC(20:2(11Z,14Z)) & 548.3707 & 6.51 & -0.6 & $\mathrm{C}_{28} \mathrm{H}_{54} \mathrm{NO}_{7} \mathrm{P}$ & $\begin{array}{l}\mathrm{M}+\mathrm{H} \\
\mathrm{M}+\mathrm{Na}\end{array}$ & 2.4 & $\uparrow$ & $\uparrow$ & $\uparrow$ \\
\hline LysoPC(20:1(11Z)) & 550.3861 & 6.99 & -1.1 & $\mathrm{C}_{28} \mathrm{H}_{56} \mathrm{NO}_{7} \mathrm{P}$ & $\mathrm{M}+\mathrm{H}$ & 2.7 & $\uparrow$ & $\uparrow$ & $\uparrow$ \\
\hline LysoPC(20:4(8Z,11Z,14Z,17Z)) & 544.3394 & 5.55 & -0.7 & $\mathrm{C}_{28} \mathrm{H}_{50} \mathrm{NO}_{7} \mathrm{P}$ & $\begin{array}{c}\mathrm{M}+\mathrm{H}- \\
\mathrm{H}_{2} \mathrm{O} \\
\mathrm{M}+\mathrm{H} \\
\mathrm{M}+\mathrm{Na}\end{array}$ & 5.1 & $\uparrow$ & $\downarrow$ & $\uparrow$ \\
\hline
\end{tabular}




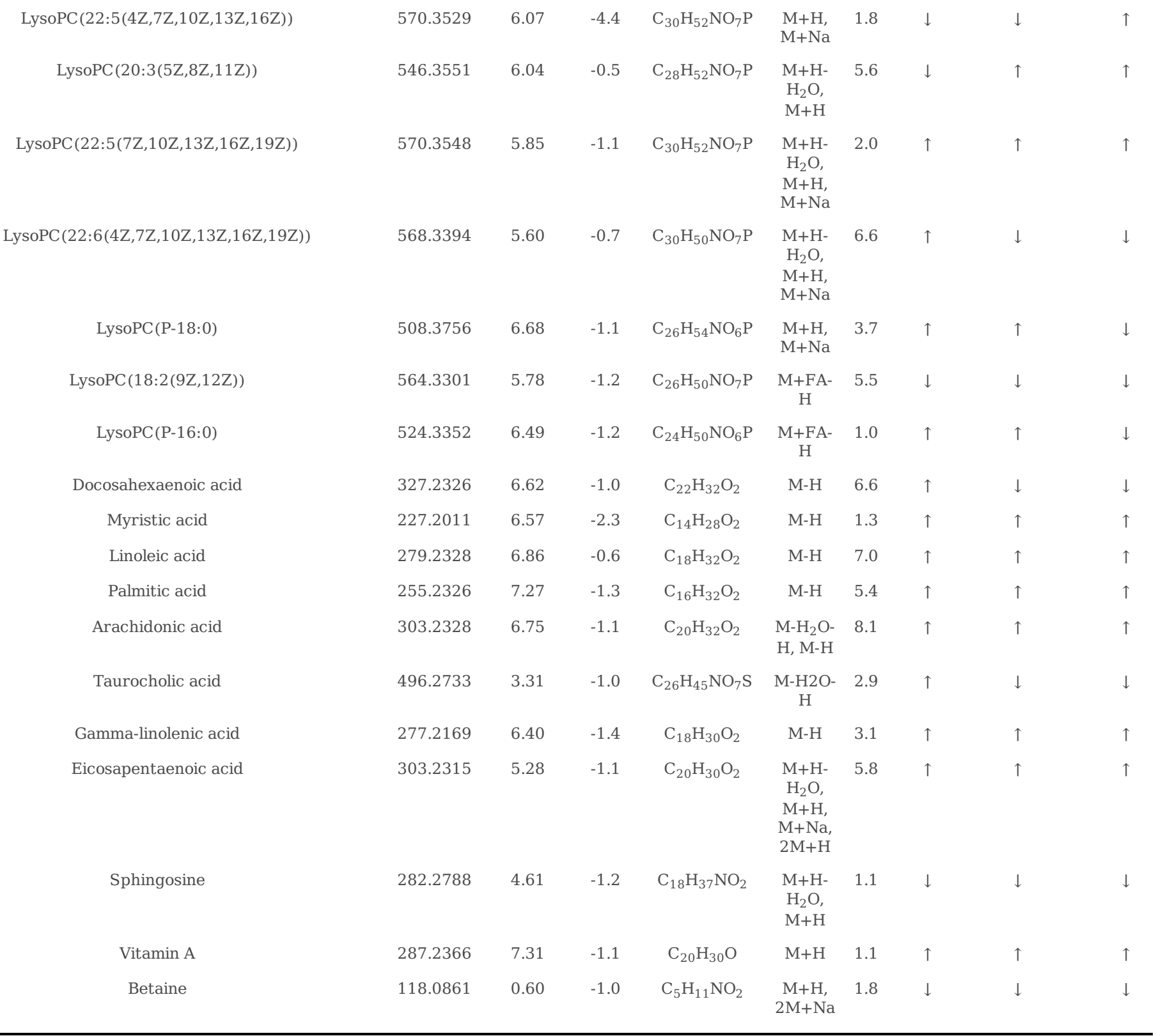




\begin{tabular}{|c|c|c|c|c|c|c|c|c|c|}
\hline Component name & MS & Observed & Mass & Formula & Adducts & VIP & \multicolumn{3}{|c|}{ Trend } \\
\hline & & & & & & & $\mathrm{NC} / \mathrm{HF}$ & $\mathrm{HF}+\mathrm{LQ} / \mathrm{HF}$ & $\mathrm{HF}+\mathrm{H}$ \\
\hline $\mathrm{PC}(20: 4(5 Z, 8 Z, 11 Z, 14 Z) / 22: 6(4 Z, 7 Z, 10 Z, 13 Z, 16 Z, 19 Z))$ & 854.5679 & 8.37 & -1.8 & $\mathrm{C}_{50} \mathrm{H}_{80} \mathrm{NO}_{8} \mathrm{P}$ & $\begin{array}{l}\mathrm{M}+\mathrm{H} \\
\mathrm{M}+\mathrm{Na}\end{array}$ & 2.3 & $\uparrow$ & $\downarrow$ & $\downarrow$ \\
\hline PC(22:6(4Z,7Z,10Z,13Z,16Z,19Z)/16:1(9Z)) & 804.5511 & 8.41 & -3.4 & $\mathrm{C}_{46} \mathrm{H}_{78} \mathrm{NO}_{8} \mathrm{P}$ & $\begin{array}{c}\mathrm{M}+\mathrm{H} \\
\mathrm{M}+\mathrm{Na}\end{array}$ & 3.2 & $\uparrow$ & $\uparrow$ & $\downarrow$ \\
\hline PC (20:5(5Z,8Z,11Z,14Z,17Z)/20:3(8Z,11Z,14Z)) & 830.5677 & 8.46 & -2.1 & $\mathrm{C}_{48} \mathrm{H}_{80} \mathrm{NO}_{8} \mathrm{P}$ & $\begin{array}{c}\mathrm{M}+\mathrm{H} \\
\mathrm{M}+\mathrm{Na}\end{array}$ & 5.1 & $\uparrow$ & $\downarrow$ & $\downarrow$ \\
\hline $\mathrm{PC}(18: 2(9 \mathrm{Z}, 12 \mathrm{Z}) / 16: 0)$ & 780.5509 & 8.94 & -0.6 & $\mathrm{C}_{42} \mathrm{H}_{80} \mathrm{NO}_{8} \mathrm{P}$ & $\mathrm{M}+\mathrm{Na}$ & 1.5 & $\downarrow$ & $\downarrow$ & $\downarrow$ \\
\hline $\mathrm{PC}(16: 0 / 18: 1(9 Z))$ & 760.5842 & 9.20 & -1.1 & $\mathrm{C}_{42} \mathrm{H}_{82} \mathrm{NO}_{8} \mathrm{P}$ & $\mathrm{M}+\mathrm{H}$ & 5.5 & $\downarrow$ & $\downarrow$ & $\downarrow$ \\
\hline Adenosine & 268.1038 & 0.80 & -0.9 & $\mathrm{C}_{10} \mathrm{H}_{13} \mathrm{~N}_{5} \mathrm{O}_{4}$ & $\mathrm{M}+\mathrm{H}$ & 1.0 & $\uparrow$ & $\downarrow$ & $\downarrow$ \\
\hline LysoPC(20:2(11Z,14Z)) & 548.3706 & 6.51 & -0.9 & $\mathrm{C}_{28} \mathrm{H}_{54} \mathrm{NO}_{7} \mathrm{P}$ & $\begin{array}{c}\mathrm{M}+\mathrm{H} \\
\mathrm{M}+\mathrm{Na}\end{array}$ & 2.1 & $\uparrow$ & $\uparrow$ & $\uparrow$ \\
\hline LysoPC(15:0) & 482.3241 & 7.02 & -0.1 & $\mathrm{C}_{23} \mathrm{H}_{48} \mathrm{NO}_{7} \mathrm{P}$ & $\begin{array}{c}\mathrm{M}+\mathrm{H} \\
\mathrm{M}+\mathrm{Na} \\
2 \mathrm{M}+\mathrm{H} \\
2 \mathrm{M}+\mathrm{Na}\end{array}$ & 3.6 & $\downarrow$ & $\downarrow$ & $\uparrow$ \\
\hline $\operatorname{SM}(d 18: 1 / 24: 1(15 Z))$ & 813.6857 & 10.05 & 1.6 & $\mathrm{C}_{47} \mathrm{H}_{93} \mathrm{~N}_{2} \mathrm{O}_{6} \mathrm{P}$ & $\begin{array}{c}\mathrm{M}+\mathrm{H} \\
\mathrm{M}+\mathrm{Na}\end{array}$ & 2.4 & $\downarrow$ & $\downarrow$ & $\downarrow$ \\
\hline Pantothenic acid & 220.1178 & 1.67 & -0.8 & $\mathrm{C}_{9} \mathrm{H}_{17} \mathrm{NO}_{5}$ & $\begin{array}{c}\mathrm{M}+\mathrm{H} \\
\mathrm{M}+\mathrm{Na}\end{array}$ & 1.2 & $\uparrow$ & $\uparrow$ & $\uparrow$ \\
\hline Pyroglutamic acid & 130.0498 & 1.15 & -0.6 & $\mathrm{C}_{5} \mathrm{H}_{7} \mathrm{NO}_{3}$ & $\mathrm{M}+\mathrm{H}$ & 1.1 & $\uparrow$ & $\downarrow$ & $\downarrow$ \\
\hline Pyrroline hydroxycarboxylic acid & 130.0499 & 0.69 & -0.1 & $\mathrm{C}_{5} \mathrm{H}_{7} \mathrm{NO}_{3}$ & $\mathrm{M}+\mathrm{H}$ & 1.5 & $\uparrow$ & $\downarrow$ & $\downarrow$ \\
\hline Docosahexaenoic acid & 329.2462 & 6.64 & -4.1 & $\mathrm{C}_{22} \mathrm{H}_{32} \mathrm{O}_{2}$ & $\begin{array}{c}\mathrm{M}+\mathrm{H} \\
\mathrm{M}+\mathrm{NH} 4 \\
\mathrm{M}+\mathrm{Na}\end{array}$ & 1.0 & $\downarrow$ & $\downarrow$ & $\downarrow$ \\
\hline Dipalmitoylphosphatidylcholine & 734.5686 & 9.22 & -1.2 & $\mathrm{C}_{40} \mathrm{H}_{80} \mathrm{NO}_{8} \mathrm{P}$ & $\mathrm{M}+\mathrm{H}$ & 2.3 & $\uparrow$ & $\downarrow$ & $\downarrow$ \\
\hline Glutathione & 308.0906 & 0.77 & -1.5 & $\mathrm{C}_{10} \mathrm{H}_{17} \mathrm{~N}_{3} \mathrm{O}_{6} \mathrm{~S}$ & $\mathrm{M}+\mathrm{H}$ & 8.8 & $\uparrow$ & $\uparrow$ & $\uparrow$ \\
\hline Oxidized glutathione & 307.0833 & 0.69 & 0.0 & $\mathrm{C}_{20} \mathrm{H}_{32} \mathrm{~N}_{6} \mathrm{O}_{12} \mathrm{~S}_{2}$ & $\begin{array}{c}\mathrm{M}+\mathrm{H} \\
\mathrm{M}+2 \mathrm{H}\end{array}$ & 4.6 & $\uparrow$ & $\downarrow$ & $\downarrow$ \\
\hline Dioleoylphosphatidylcholine & 786.5996 & 9.25 & -1.5 & $\mathrm{C}_{44} \mathrm{H}_{84} \mathrm{NO}_{8} \mathrm{P}$ & $\mathrm{M}+\mathrm{H}$ & 6.0 & $\downarrow$ & $\downarrow$ & $\downarrow$ \\
\hline FAD & 786.1630 & 1.70 & -1.8 & $\mathrm{C}_{27} \mathrm{H}_{33} \mathrm{~N}_{9} \mathrm{O}_{15} \mathrm{P}_{2}$ & $\mathrm{M}+\mathrm{H}$ & 1.1 & $\uparrow$ & $\uparrow$ & $\downarrow$ \\
\hline Niacinamide & 123.0553 & 0.67 & 0.1 & $\mathrm{C}_{6} \mathrm{H}_{6} \mathrm{~N}_{2} \mathrm{O}$ & $\mathrm{M}+\mathrm{H}$ & 1.2 & $\uparrow$ & $\downarrow$ & $\downarrow$ \\
\hline Pantetheine $4^{\prime}$-phosphate & 359.1033 & 1.66 & -0.9 & $\mathrm{C}_{11} \mathrm{H}_{23} \mathrm{~N}_{2} \mathrm{O}_{7} \mathrm{P}_{\mathrm{S}}$ & $\begin{array}{c}\mathrm{M}+\mathrm{H} \\
\mathrm{M}+\mathrm{Na} \\
2 \mathrm{M}+\mathrm{H} \\
2 \mathrm{M}+\mathrm{Na}\end{array}$ & 2.7 & $\uparrow$ & $\uparrow$ & $\uparrow$ \\
\hline Inosine & 267.0734 & 1.54 & -0.5 & $\mathrm{C}_{10} \mathrm{H}_{12} \mathrm{~N}_{4} \mathrm{O}_{5}$ & $\mathrm{M}-\mathrm{H}$ & 3.9 & $\uparrow$ & $\downarrow$ & $\downarrow$ \\
\hline Glycerol 3-phosphate & 171.0063 & 0.61 & -0.6 & $\mathrm{C}_{3} \mathrm{H}_{9} \mathrm{O}_{6} \mathrm{P}$ & $\mathrm{M}-\mathrm{H}$ & 1.3 & $\uparrow$ & $\uparrow$ & $\uparrow$ \\
\hline
\end{tabular}


A

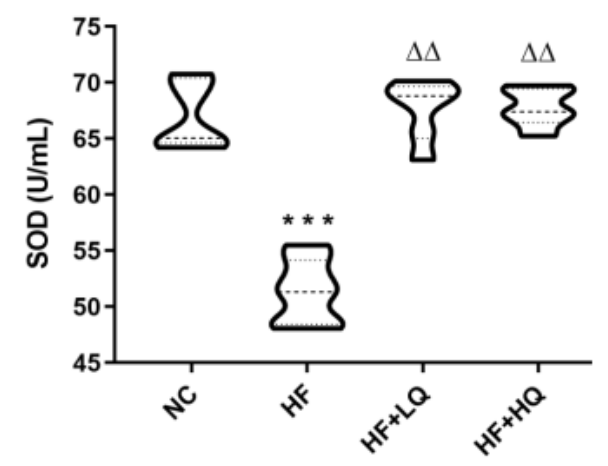

C

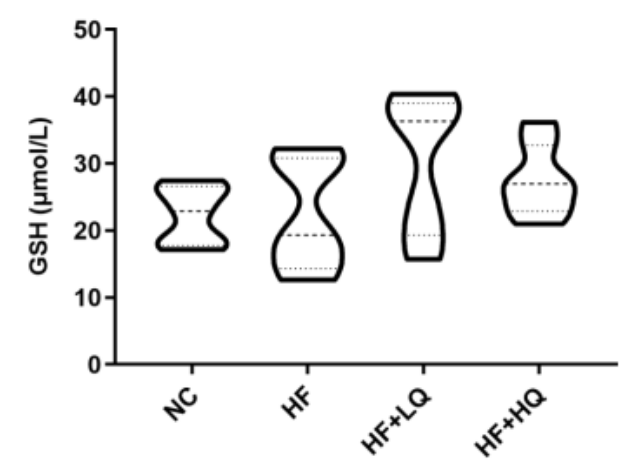

B

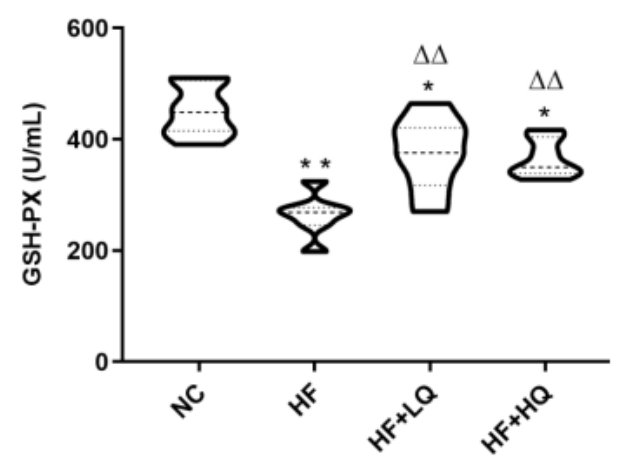

D

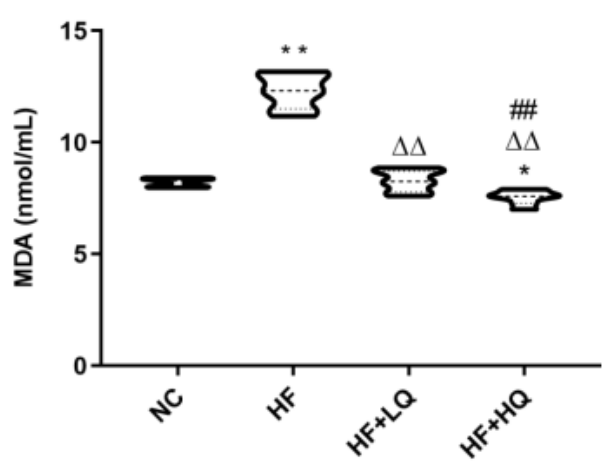

Figure 2

Effects of quinoa on serum anti-oxidative parameters of rats fed on a high-fat diet. A. SOD activity. B. GSH-PX activity. C. GSH level. D. MDA level. NC: normal chow diet control group ( $n=7)$; HF: a high-fat diet group ( $n=7)$; HF + LQ: low-dose quinoa diet group $(n=7)$; HF + HQ: a high-dose quinoa diet group ( $n=7)$. The rats in the NC group were fed a standard diet, and those in the other three groups were fed an HF diet. Simultaneously, the low-dose quinoa feed (HF + LQ) was added to $9 \%$ of quinoa (equivalent to $100 \mathrm{~g}$ of daily human intake), and the high-dose quinoa feed (HF $+\mathrm{HQ}$ ) was added to $27 \%$ of quinoa (equivalent to $300 \mathrm{~g}$ of daily human intake). ${ }^{\star} \mathrm{p}<0.05 \mathrm{vs}$. the NC group, ${ }^{\star \star} \mathrm{p}<0.01 \mathrm{vs}$. the NC group; $\Delta \mathrm{p}<0.05 \mathrm{vs}$. the HF group, $\Delta \Delta \mathrm{p}<0.01$ vs. the HF group; \#p $<0.05$ vs. the HF + LQ group; \#\#p < 0.01 vs. the HF + LQ group. The bars show the mean values with standard deviations. 


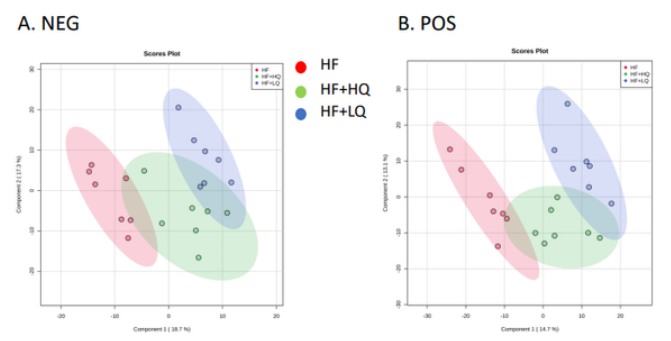

c.
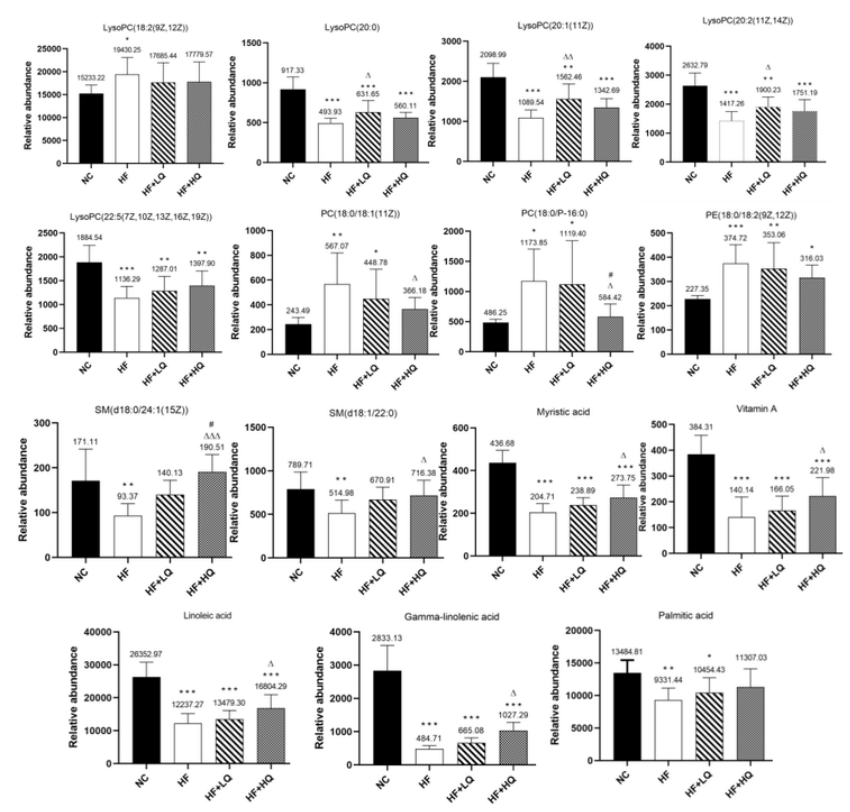

\section{Figure 3}

Untargeted serum metabolomics analysis of rats fed on a high-fat diet. A and B. PLS-DA (partial least squares discrimination analysis) score plot under negative and positive ion modes for serum metabolites obtained by UPLC-QTOF-MSE. C. Partial differential metabolites in the serum. NC: normal chow diet control group ( $n=7)$; HF: a high-fat diet group ( $n=7)$; HF + LQ: low-dose quinoa diet group $(n=7)$; HF + HQ: a high-dose quinoa diet group ( $n=7)$. The rats in the NC group were fed a standard diet, and those in the other three groups were fed an HF diet. Simultaneously, the low-dose quinoa feed (HF + LQ) was added to $9 \%$ of quinoa (equivalent to $100 \mathrm{~g}$ of daily human intake), and the high-dose quinoa feed (HF $+\mathrm{HQ}$ ) was added to $27 \%$ of quinoa (equivalent to $300 \mathrm{~g}$ of daily human intake). ${ }^{*} \mathrm{p}<0.05$ vs. the NC group, ${ }^{*} \mathrm{p} p<0.01$ vs. the NC group; $\Delta \mathrm{p}<0.05$ vs. the HF group, $\Delta \Delta \mathrm{p}<0.01$ vs. the HF group; \#p $<0.05$ vs. the HF $+\mathrm{LQ}$ group; \#\#p 0.01 vs. the HF + LQ group. The bars show the mean values with standard deviations. 


\section{Hepatic tissue}

\section{A. NEG}

\section{B. POS}
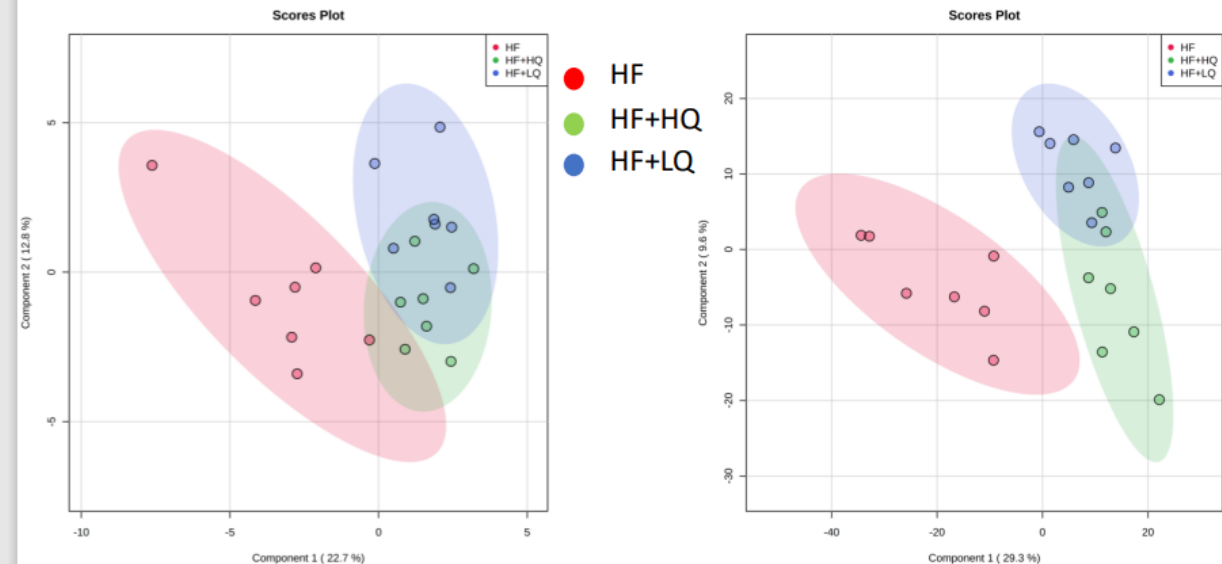

C.
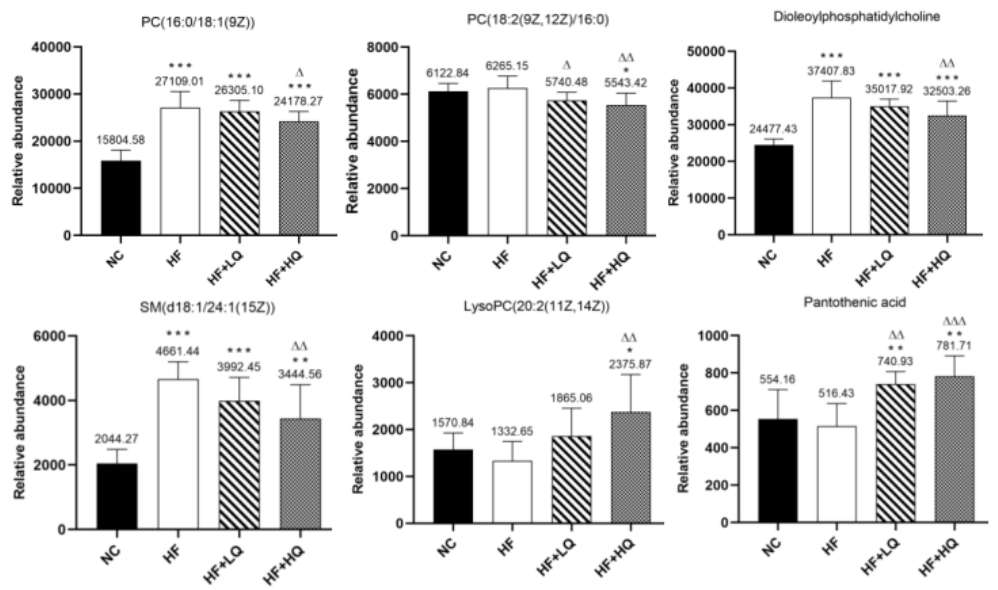

\section{Figure 4}

Untargeted hepatic metabolomics analysis of rats fed on a high-fat diet. A and B. PLS-DA (partial least squares discrimination analysis) score plot under negative and positive ion modes for the hepatic metabolites obtained by UPLC-QTOF-MSE. C. Partial differential metabolites in the liver. NC: normal chow diet control group ( $n=7)$; HF: a high-fat diet group ( $n=7)$; HF + LQ: low-dose quinoa diet group $(n=7)$; HF + HQ: a high-dose quinoa diet group ( $n=7)$. The rats in the NC group were fed a standard diet, and those in the other three groups were fed an HF diet. Simultaneously, the low-dose quinoa feed (HF + LQ) was added to $9 \%$ of quinoa (equivalent to $100 \mathrm{~g}$ of daily human intake), and the high-dose quinoa feed (HF $+\mathrm{HQ}$ ) was added to $27 \%$ of quinoa (equivalent to $300 \mathrm{~g}$ of daily human intake). ${ }^{*} \mathrm{p}<0.05$ vs. the NC group, ${ }^{*} \mathrm{p} p<0.01$ vs. the NC group; $\Delta \mathrm{p}<0.05$ vs. the HF group, $\Delta \Delta \mathrm{p}<0.01$ vs. the HF group; \#p $<0.05$ vs. the HF $+\mathrm{LQ}$ group; \#\#p 0.01 vs. the HF + LQ group. The bars show the mean values with standard deviations. 

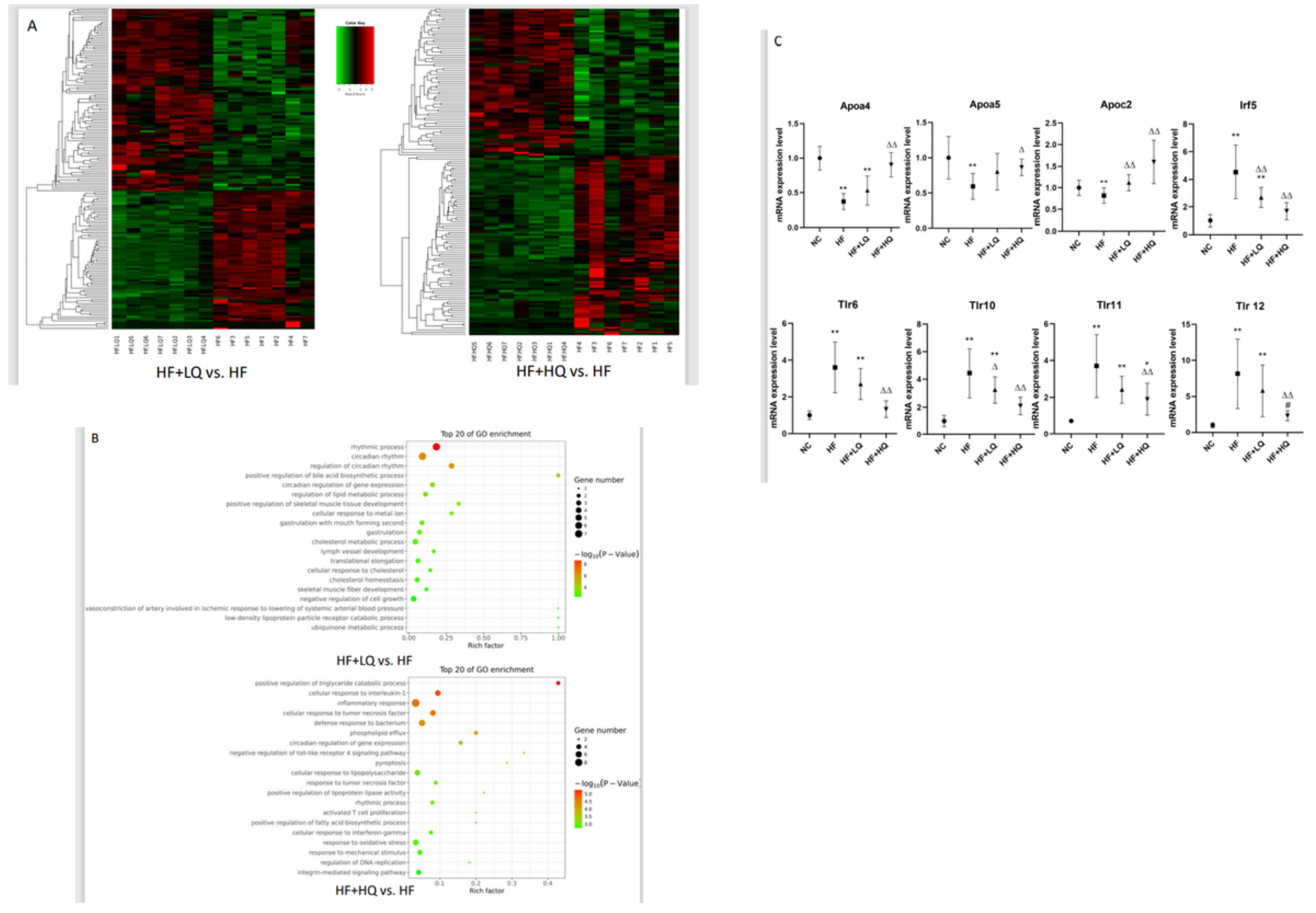

\section{Figure 5}

Effects of quinoa on hepatic gene expression profile at mRNA levels of rats fed on a high-fat diet. A. Heatmap analysis; B. GO analysis and clustering analysis; C. Verification of partial gene mRNA expression level using qRT-PCR. NC: normal chow diet control group $(n=7)$; HF: a high-fat diet group $(n=7)$; HF + LQ: lowdose quinoa diet group $(n=7)$; HF + HQ: a high-dose quinoa diet group $(n=7)$. The rats in the NC group were fed a standard diet, and those in the other groups were fed an HF diet. Simultaneously, the low-dose quinoa feed (HF + LQ) was added to $9 \%$ of quinoa (equivalent to $100 \mathrm{~g}$ of daily human intake), and the highdose quinoa feed (HF + HQ) was added to $27 \%$ of quinoa (equivalent to $300 \mathrm{~g}$ of daily human intake).

\section{Supplementary Files}

This is a list of supplementary files associated with this preprint. Click to download.

- SupplementaryTable12.docx 